\title{
Effects of Repetition and Foreknowledge in Task-Set Reconfiguration
}

\author{
Myeong-Ho Sohn and Richard A. Carlson \\ Pennsylvania State University, University Park Campus
}

\begin{abstract}
To examine the roles of executive control and automatic activation in task switching, we manipulated foreknowledge as well as task transitions. In Experiments 1 and 2, performance with foreknowledge was faster than performance with no foreknowledge, but the amount of switch cost did not depend on foreknowledge. This result suggests that switch costs primarily reflect persisting activation rather than inadequate preparation. In Experiment 3, switch cost was greater with foreknowledge about task transition alone than with foreknowledge about both task transition and identity, suggesting that foreknowledge about specific task identity did allow preparation for a switched task. We argue that task repetition and foreknowledge effects are independent. Although foreknowledge allows preparation for both repeated and switched tasks, repeating the same task has benefits over task switching regardless of foreknowledge.
\end{abstract}

People perform most of their cognitive activities by deliberately applying their intention to achieve the goal that is relevant at the moment. Forming an effective intention to perform a particular task can be described as adopting a task set (Rogers \& Monsell, 1995). A task set specifies the relevant response or action to a target stimulus in a given situation, and configuring the cognitive system for a new task is called task-set reconfiguration (Rogers \& Monsell, 1995). The classical view is that there are two control mechanisms in human cognition: executive control and automatic control. Although various pairs of terms have been used, these mechanisms can be characterized by their differing dependence on internal representation of the current goal, when applied to task-set reconfiguration.

Executive control is endogenous, goal-directed, intentional, and voluntary, reflecting the internal representation of a current goal. For example, foreknowledge that specifies a goal allows advance preparation or reconfiguration for a task set even if the objects to be processed are not yet available (Carlson \& Lundy, 1992; Sohn \& Carlson, 1998). The executive control mechanism has been identified as a central executive (Baddeley, 1986), a supervisory attentional system (Norman \& Shallice, 1980; Shallice, 1994), executive function (Logan, 1985), or controlled processing (Schneider \& Shiffrin, 1977). Alternatively, automatic control is

Myeong-Ho Sohn and Richard A. Carlson, Department of Psychology, Pennsylvania State University, University Park Campus.

This work was supported by the Weiss Dissertation Fellowship from the Weiss Scholars Program, Pennsylvania State University, University Park. The research reported in this article was carried out as part of Myeong-Ho Sohn's doctoral dissertation submitted to the Pennsylvania State University, University Park.

We thank Roger Downs, Lori Forlizzi, Dani Gopher, Frank Lee, Sander Los, Nachshon Meiran, Cathleen Moore, Tram Neill, David Rosenbaum, and an anonymous reviewer for their helpful comments on earlier versions of this article.

Correspondence concerning this article should be addressed to Myeong-Ho Sohn, who is now at BH 345E, Department of Psychology, Carnegie Mellon University, Pittsburgh, Pennsylvania 15213. Electronic mail may be sent to mhsohn $+@$ andrew.cmu.edu. driven by an external stimulus or event and does not necessarily reflect the current goal. For example, Neely (1977) showed that time course of priming effect did not depend on foreknowledge. This is because the activation change resulting from priming is not under conscious control. Another example of automatic control is "utilization behavior" of frontal lobe patients. When these patients encounter everyday objects, they cannot help but perform complete action sequences characteristically associated with them, even though the actions are not intended or desirable (Lhermitte, 1983; Shallice, Burgess, Schon, \& Baxter, 1989). This demonstrates that the automatic control may have its effect regardless of an individual's intention. The automatic control mechanism has been described as automatic processing (Schneider \& Shiffrin, 1977) or contention scheduling (Norman \& Shallice, 1980).

The purpose of the current study is to investigate how executive and automatic control mechanisms operate when people have to rapidly reconfigure their mental processes for a cognitive task set. We focus on the effect of foreknowledge as the source of executive control and persisting activation as the source of automatic control. Our hypothesis is that, although both executive control and automatic control are necessary for the most efficient task-set reconfiguration, their effects may be independent. This hypothesis is based on a common implication of previous studies on executive and automatic control: Activation change due to automatic mechanisms is not directly under the control of executive mechanism, while executive mechanisms can also increase activation (Neely, 1977; Schneider \& Shiffrin, 1977; Posner \& Snyder, 1975). Specifically, we expect that foreknowledge should allow endogenous preparation of a task set even without external objects to process, regardless of whether the same task is repeated or not. Also, repeating the same task should benefit performance regardless of whether foreknowledge is available regarding a specific task set. In the following section, we briefly describe the phenomenon of interest which is called task-switch cost, and then introduce our methodology.

\section{Task Switch Cost}

The task-switching paradigm has been extensively used to study executive and automatic control. In this paradigm, participants 
rapidly repeat the same task or alternate between different tasks. Typically, several stimuli are presented in a sequence, and each stimulus requires a separate response. A response-to-stimulus interval (RSI) separates the previous response and the current stimulus. A stimulus consists of two elements, each of which is associated with a different task set. For example, the stimulus might consist of one letter and one digit, "G7," and a participant is instructed to perform one of two tasks (Rogers \& Monsell, 1995). In the letter task (Task L), one must decide whether the letter is a consonant or a vowel while ignoring the digit and, in the digit task (Task D), whether the digit is an even number or an odd number while ignoring the letter. Which task to perform is indicated by an external cue, such as the location of the stimuli, or by a preinstructed sequence. If the current task set in a sequence is the same as the previous one, it is called a task repetition, and if not, it is called a task switch. Because the stimulus presentation is controlled by an RSI, the imperative stimulus for a task will be available only after the goal for the previous task has been achieved to some extent (e.g., after a response for that previous task is generated). The imperative stimulus indicates both the relevant task set and the objects to be judged or processed by the task set and provides a signal to begin the task.

A typical result is that the latencies are longer and sometimes the error rates are higher for task switches than for task repetitions (Allport, Styles, \& Hsieh, 1994; Gopher, Armony, \& Greenshpan, 1998; Jersild, 1927; Rogers \& Monsell, 1995; Spector \& Biederman, 1976). The decrement in performance from task switching is called a switch cost. It should be noted that in most of the cited studies there were two tasks and the task transition-whether to switch or to repeat--was predictable. Hence, if task-set reconfiguration is completely under executive control, the cost should disappear with a sufficiently long RSI to prepare for the reconfiguration. However, although the switch cost was reduced as the RSI increased, it was not eliminated even with a very long RSI (Allport et al., 1994; Rogers \& Monsell, 1995). This residual switch cost implies that there are some constraints on executive control when a person rapidly adopts different task sets, one after another.

Alport et al. (1994) argued that the source of the residual switch cost is a kind of inertia. According to their task-set inertia hypothesis, the reduction of switch cost during RSI reflects decaying activation of a previous task set, which implies that switch cost may, in fact, reflect the benefit of repeating the same task. The activation of a just-performed task set is not directly under executive control in that, once a task is performed, the activation does not diminish completely even when participants know that the task is no longer relevant. This residual activation may facilitate performance of the repeated task but interfere with performance of a switched task. The interference caused by persisting activation from a previous task set is not under control of participants, reflecting instead an automatic or exogenous source of control. Although this interference may reduce as a function of time, its complete reduction may depend on arrival of a new stimulus, providing the source of the residual switch cost.

Rogers and Monsell (1995) questioned whether the reduction of switch cost with increasing RSI actually reflects decay of activation of a previous task set and whether the residual switch cost reflects inertia. In their experiments, the reduction of switch cost as a function of RSI was obtained only when the RSI was blocked but not when it varied within blocks. This suggests that preparation during the RSI is related to the onset of the next stimulus, because, if switch cost reflects diminishing activation from the previous task set, then it should decline as time goes by, regardless of whether the RSI was predictable. Similarly, Meiran (1996) found switch cost reduction as a function of cue-to-target interval between a cue for a stimulus and actual arrival of the stimulus. Because he used a fixed RSI in which the cue-to-target interval was embedded, Meiran argued that the reduction of the switch cost cannot be attributed to reduction in interference from a previous task set. An implication of both studies is that the switch cost does not result from automatically decaying activation from the previous task set. Instead, we suggest, it may be related to the preparation for a new task, such as when to execute the relevant task. For example, foreknowledge about when to execute a task may trigger the increase of activation of the relevant task set, and the reduction of the switch cost with increasing RSI may reflect this increase in activation.

According to Rogers and Monsell's (1995) stimulus-cued completion hypothesis, the completion of task-set reconfiguration is triggered only by a task-associated stimulus and must wait on its presentation. Like Allport et al. (1994), Rogers and Monsell also suggested that there may be two components to the switch cost: one associated with endogenous preparation through executive control and the other associated with exogenous completion of preparation through automatic control. Interestingly, they argued that neither of these components is associated with proactive interference by the previous task set but instead are associated with the preparation of a new task set. That is, the reduction of the switch cost with increasing RSI may reflect increasing level of preparation of a new task set in advance of an external stimulus. For example, Rogers and Monsell (1995) implied that the residual switch cost reflected continuing changes in activation after a stimulus for a new task is presented. This change may be related to a previous task set, which shares a common stimulus with the new task set, and this is similar to Allport et al.'s (1994) inertia hypothesis. However, Rogers and Monsell proposed different ideas on the time course of the residual switch cost from Allport et al.; this is discussed more later. A more important difference is that the implications of Rogers and Monsell's results contrast with Allport et al.'s (1994) view concerning the nature of executive preparation of a new task set before stimulus presentation.

De Jong (in press; De Jong, Berendsen, \& Cools, 1999) proposed a mixture-probability model of preparation for the task switch cost. According to him, participants may more likely fail to prepare for task switches than for task repetitions. However, once task reconfiguration has been completed, performing a switched task does not require any additional processes compared with performing a repeated task. In support of his view, he analyzed the cumulative distribution of response times of task switch and task repetition. His assumption was that faster latencies may reflect successfully prepared trials and slower trials may reflect failed preparation. His analysis revealed that, with sufficiently long time permitted for preparation, fast switch latencies were almost identical to fast repetition latencies, supporting his hypothesis. In contrast, repetition latencies did not differ regardless of RSI, suggesting that preparation for repetition might be always nearly perfect. De Jong thus assumed that switch and repetition trials differ in the demand for preparation and that preparation sometimes fails on switch trials. Although De Jong does not commit 
himself to a specific model of task switch, our interpretation is that his result is consistent with the position that inadequate preparation is the source of switch cost. That is, less-than-perfect preparation may not be a problem at the level of individual trials. However, at a global level, some proportion of switch trials may not be prepared properly, resulting in switch cost.

The purpose of the current study is to investigate the nature of the switch cost in the context of foreknowledge. According to Allport et al. (1994), the switch cost reflects the persisting activation of a previous task. If the previous task is repeated, this persisting activation may facilitate performance. However, if the current task is different from the previous one, the persisting activation will interfere with the performance of the current task. According to Rogers and Monsell (1995), the switch cost reflects inadequate preparation for a new task set. When a task is performed a second time, preparation for the task set is complete, because it has just been performed. However, the first time the task is performed, completion of task-set reconfiguration depends on arrival of an external stimulus that supports the next task set. That is, even with foreknowledge, the preparation for a switch is less than complete, unlike the preparation for a repetition. These two views on switch costs have different implications for foreknowledge. If switch costs depend on persisting activation, the effect should be observed regardless of foreknowledge because the activation is not under executive control. However, if switch costs depend on differential preparation, the effect should be observed only with foreknowledge, which allows preparation. With no foreknowledge, there should be no effect of differential preparation because neither a repetition nor a switch can be prepared.

\section{Current Study}

We ask two questions in this study. First, are the repetition effect and preparation resulting from foreknowledge separable processes that both occur in a task switch situation? Second, how do they have their effects, especially before an external stimulus for a new task is presented? Several aspects of previous studies make it hard to address these questions on the basis of prior results. First, in many task-switching studies already mentioned, foreknowledge about task-transition information (e.g., whether to switch or to repeat) was available. For example, task transition was blocked (Allport et al., 1994), the order of transitions was periodically repeated (Rogers \& Monsell, 1995), or there was a valid cue for a task transition before the stimulus for the next task was presented (Meiran, 1996). In contrast, we examined the difference between task repetitions and task switches with no foreknowledge as well as with foreknowledge. This was necessary because, by hypothesis, preparation can be affected by foreknowledge but persisting activation cannot. Participants cannot depend on executive control to prepare for a new task set endogenously without task identity foreknowledge (unless they guess which task set will be relevant, a possibility considered later). However, even without such foreknowledge, participants could benefit from task repetition if the time course of activation is automatic.

Second, in previous studies, there were always two task sets within a block or within an experiment. When there are only two task sets and the transition type is known, task-identity information is confounded with task-transition information. We examined the switch cost with three task sets as well as two task sets. Using three tasks allows task transition information to be available without necessarily conveying task identity information. When foreknowledge carries only task transition information but not task identity information, specific preparation for a new task cannot be initiated. Therefore, it is possible to examine whether the switch cost involves preparation only for switching from a previously performed task or increasing preparation for a new task, or both. If the switch cost simply reflects switching from the previous task, the amount of switch cost should not depend on either partial or full foreknowledge. This is because the decay of activation should be the same regardless of the extent of foreknowledge. However, if executive preparation for a specific new task is also operating, the switch cost should depend on whether foreknowledge about specific task identity is available or not.

There is another methodological limitation in previous taskswitching paradigms that makes it difficult to address the question of preparation and repetition. In many task-switching studies with a few exceptions, a predictable task transition was embedded in a fairly long sequence of steps. In the alternating-runs paradigm (Experiments 1-4, Rogers \& Monsell, 1995), for example, a pair of Task L (letter task) alternated with a pair of Task D (digit task) periodically. Therefore, a repeated task was always followed by a switched task (i.e., . . LLDD . . ), and a switched task was always followed by a repeated task (i.e., . . LLDD ...). Consequently, a repeated task was performed while a task switch was prepared, and a switched task was performed while a task repetition was prepared. Therefore, it is not clear whether processing time for a certain task is related entirely to a task switch or a task repetition, because concurrent performance of the current task and preparation for the following task might affect response time. In the current study, we used a modified task-switching paradigm in which sequences contain only two tasks. ${ }^{1}$ Figure 1 shows a schematic of a typical trial. Because the focus of the current study is on the role of foreknowledge, and not of RSI, a response for Task 1 and a stimulus for Task 2 were separated by a fixed RSI of 1,000 $\mathrm{ms}$, which should be long enough to allow endogenous preparation (Rogers \& Monsell, 1995). The task set identity of each task was indicated by the color of the stimulus.

\section{Overview and Predictions}

In Experiments I and 2, half of participants had full foreknowledge of the task transition and identity of Task 2 , and the other half

\footnotetext{
${ }^{1}$ It should be noted that we are not the first investigators who have adopted a shorter sequence paradigm. For example, Allport et al. (1994) used a similar paradigm (Experiment 5). However, there is one critical difference between their paradigm and ours. In Allport et al.'s study, the identity of both the first and the second tasks was always known globally for a block of trials. To illustrate with the tasks we used, either an LL-LL-LL-LL-LL sequence or DD-DD-DD-DD-DD sequence could have been used in a repetition list, and either LD-LD-LD-LD-LD sequence or DL-DL-DL-DL-DL sequence in a switch list. Because the repetition list contains only one task while the switch list contains two tasks, it is not easy to attribute the difference between switch and repetition solely to the transition difference. For example, participants might have had to maintain a higher working memory load in the switch list as well as face a greater processing load to switch between two different task sets. In our paradigm, however, there were always the same number of task sets in a block regardless of the foreknowledge available in the block.
} 


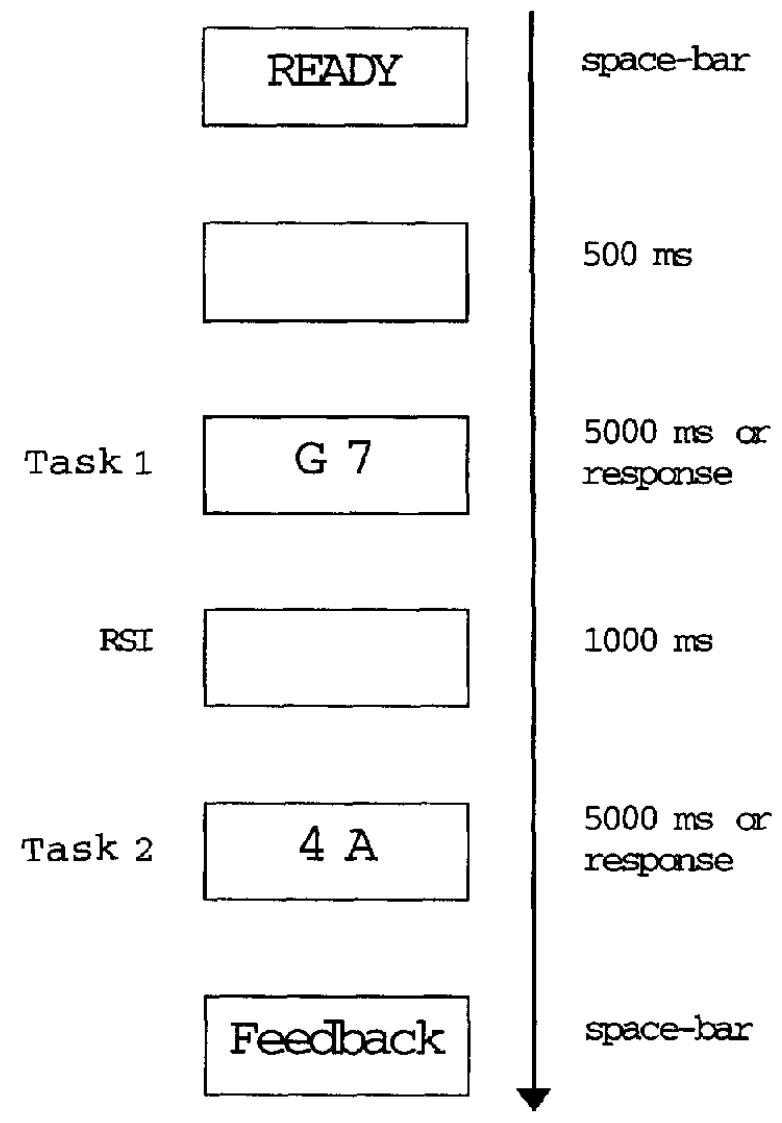

Figure 1. A schematic of a typical trial in Experiments 1 and 2.

had no foreknowledge at all. To allow comparison with previous studies, these two experiments adopted only two task sets. Our main concern is whether foreknowledge would result in any benefit in Task 2 performance, and if so, whether the benefit of foreknowledge would interact with the cost of switching.

First, consider the predictions based on the assumption that full foreknowledge allows advance preparation for Task 2 . In this case, Task 2 latency with foreknowledge should be faster than Task 2 latency with no foreknowledge. When foreknowledge about task identity is available, participants will either actively maintain the current task set with a repetition or adopt another task set with a switch. In contrast, without such foreknowledge, participants may prepare neither task or prepare one of the tasks at random. In either case, there should be a decrement in performance compared with preparation on the basis of foreknowledge. Therefore, Task 2 should be faster with foreknowledge than with no foreknowledge.

Second, consider predictions based on the further assumption that the effects of persisting activation and preparation are independent. If they are independent, the magnitude of the switch cost should be the same with or without foreknowledge. If the persisting activation of Task 1 is the source of switch cost and this activation is not under executive control (Allport et al., 1994), persisting activation should facilitate repeated tasks and inhibit switched tasks, regardless of foreknowledge. Although foreknowledge may facilitate performance in general, it should occur for both repetitions and switches.
Third, suppose that persisting activation and preparation are not independent and that inadequate preparation is the source of switch cost when preparation is permitted for both repetition and switch cases. Then the magnitude of the switch cost would depend on foreknowledge. With foreknowledge, although participants can prepare for both repetitions and switches, preparation for switches would be inadequate because a stimulus is required to complete the preparation. With no foreknowledge, however, the extent of the preparation for repetition and switch should be equivalent. Hence, the difference between repetition and switch with no foreknowledge should reflect only persisting activation, whereas the difference with foreknowledge will reflect both persisting activation and different degrees of preparation.

These predictions assume that participants will prepare for neither switched nor repeated task set with no foreknowledge. Alternatively, they can prepare for either switched or repeated task set at random. When adopted on every trial, this strategy benefits a random half of repeated and switched trials, while hurting the other half. For the half of trials with no foreknowledge on which participant's guess was correct, the switch cost would be comparable to the switch cost with foreknowledge. However, for the other random half of trials on which the guess was wrong, the switch cost should be relatively smaller. Therefore, whichever strategy is adopted, if the persisting activation from the previous task is not considered, the switch cost should be greater with foreknowledge than with no foreknowledge.

Ruthruff, Remington, and Johnston (1996) addressed very similar questions regarding the roles of foreknowledge and task repetition. Using a paradigm similar to that of Rogers and Monsell (1995), they reported that the benefit of task repetition over task switch depended on the extent of expectancy of the next task set. In their Experiments 1 to 3, the switch cost was greater when a certain task set was highly but not completely predictable than when the task set was less predictable. However, in their Experiment 4 , when the task set was completely random, the switch cost was comparable to when the task set was completely predictable. Although we regard their Experiment 4 result as supporting the independence of persisting activation and foreknowledge effects, they argued that the switch cost may not be completely automatic because the amount of benefit seemed to be sensitive to probability. We discuss this difference in interpretation later.

To anticipate, results from Experiments 1 and 2 showed equivalent effects of foreknowledge for repetition and switch trials, suggesting that the extent of advance preparation for both trial types on the basis of foreknowledge was equivalent. In Experiments 1 and 2, however, it is not clear whether effects of foreknowledge reflect preparation for a specific task set or only for the need to switch tasks, because only two tasks were used. Experiment 3 examined the nature of preparation: whether the preparation for a task switch is specific to a task set or general for task switching. Participants performed task-switch and task-repetition trials with three tasks. Half of participants had full foreknowledge, specifying both task transition and task identity. The other half had only partial foreknowledge, which provided task transition foreknowledge, whether to repeat or switch, but not foreknowledge of Task 2 identity. Therefore, the difference between full and partial foreknowledge is in the amount of information they provide for a task switch. This partial foreknowledge provides task transition but not task identity information. If preparation for a task switch is for 
task transitions in general, the Task 2 latency would not depend on whether foreknowledge is partial or full. However, if preparation is task specific, then Task 2 latency should be faster with full foreknowledge than with partial foreknowledge.

\section{Experiment 1}

Experiment 1 had two purposes: to examine whether foreknowledge allows endogenous preparation for Task 2 and to examine the nature of the process that is reflected in the switch cost. Table 1 illustrates the foreknowledge manipulations in Experiment 1. In the foreknowledge condition, participants performed only switch trials in some blocks and only repetition trials in other blocks. They received explicit instructions about the organization of each block. In contrast, participants in the no-foreknowledge condition performed both task repetitions and task switches randomly mixed within each block. They received explicit instructions that the two types of transition would appear randomly in each block.

Our main concern is the theoretical implications of preparation and repetition effects on Task 2 latency, and we focus on Task 2 latency as a function of within-trial transition and foreknowledge. Because the full foreknowledge available in the foreknowledge condition should allow advance preparation, Task 2 latency in this condition should be faster than Task 2 latency in the noforeknowledge condition. The residual switch cost obtained in previous studies implies that, even when task-set reconfiguration can be endogenously prepared before Task 2 stimulus presentation, the completion of task-set reconfiguration requires an external stimulus (Rogers \& Monsell, 1995).

If this switch cost reflects persisting activation of Task 1 , then Task 2 switch cost in the foreknowledge condition should not differ from that of the no-foreknowledge condition. This is because the activation change does not depend on foreknowledge. In contrast, if switch cost reflects exogenous completion of preparation for a new task (Rogers \& Monsell, 1995) or probabilistic failure to prepare (De Jong, in press; De Jong et al., 1999), then the amount of switch cost should be greater with foreknowledge than with no foreknowledge. With no foreknowledge, the extent of preparation for repetition and switch should be equivalent. However, with foreknowledge, preparation for a repeated task would be complete, whereas preparation for a switched task may not be. Therefore, Task 2 switch cost in the foreknowledge condition should be greater than in the no-foreknowledge condition.

There is also a question whether Task 1 performance is affected by foreknowledge. With foreknowledge, Task 1 may be performed

Table 1

An Illustration of the Foreknowledge Manipulation in Experiment 1

\begin{tabular}{llll}
\hline \multicolumn{1}{c}{ Condition } & Type of block & Task 1 & Task 2 \\
\hline Foreknowledge & Repetition block & Letter & Letter \\
& & Digit & Digit \\
& Switch block & Digit & Letter \\
& & Letter & Digit \\
No foreknowledge & Mixed block & Letter & Letter \\
& & Letter & Digit \\
& & Digit & Letter \\
& & Digit & Digit \\
\hline
\end{tabular}

while preparation for the next task is underway. With no foreknowledge, this may not be the case. Therefore, if participants with foreknowledge prepared Task 2 while performing Task 1 , then this should slow down Task 1 latency compared with those cases with no foreknowledge. We attempted to address this question by looking at intertrial sequential effects. One drawback of our paradigm is that, because of the short sequences, intertrial transitions as well as within-trial transitions may affect performance. With or without foreknowledge, the relationship between Task 1 and previous Task 2 is random. However, the context for intertrial carryover depends on whether or not there is foreknowledge. For example, consider two consecutive trials in the repetition block with foreknowledge. On some of these trials, Task 2 could be the fourth consecutive repetition of that task set (e.g., LL-LL or DD-DD). One consequence of our foreknowledge manipulation is that this type of intertrial transition occurred most frequently in the repetition blocks with foreknowledge, never in the switch blocks with foreknowledge, and with intermediate frequency in noforeknowledge conditions. Table 2 illustrates the possible intertrial relations in Experiments 1 and 2. To examine this potential confound, we analyzed the effect of intertrial sequences on both Task 1 and Task 2 latencies. To anticipate, intertrial transition does have some effects on Task 1 and Task 2 performance but does not compromise the interpretation of within-trial transition and foreknowledge effects on Task 2 performance.

\section{Method}

Task and equipment. Stimuli were generated using Micro Experimental Lab (MEL) system software (Psychology Software Tools, Pittsburgh, PA), and the timing was controlled by an IBM-compatible PC. Stimuli and tasks were similar to those used in Rogers and Monsell (1995). A stimulus consisted of one letter and one digit. The letter was one of four consonants (G, K, M, and R) or four vowels (A, E, I, and U). The digit was one of four even numbers $(2,4,6$, and 8$)$ or four odd numbers $(3,5,7$, and 9$)$. For half of trials, the left-right order of stimulus elements was letter-digit, and this order was reversed for the other half of trials. A participant's task was to make a decision about whether the letter was a consonant or a vowel, letter task (Task L), or whether the digit was an even number or an odd number, digit task (Task D). The task identity was indicated by the color of the stimulus. For example, if the stimulus was in red (MEL color code 13), then the task was the letter task; if the stimulus was in green (MEL color code 2), then the task was the digit task. Task-color mapping was counterbalanced across participants.

Procedure and design. As shown in Figure 1, every trial began with the "READY" signal at the center of a computer screen. A participant initiated a trial by pressing the space bar. A blank screen then replaced the READY signal for $500 \mathrm{~ms}$, followed by a stimulus for Task 1 . The stimulus remained on the screen until the participant pressed a response key or until $5,000 \mathrm{~ms}$ had elapsed. After an RSI of $1,000 \mathrm{~ms}$, a stimulus for Task 2 appeared and remained on the screen until the participant pressed a response key or until 5,000 ms had elapsed. The "even" and "consonant" responses were assigned to the same response key and the "odd" and "vowel" responses were assigned to another response key. The mapping between responses and response keys was counterbalanced across participants. At the end of each trial, feedback about accuracy and latency for both steps was given.

At the beginning of each experimental session, there was a practice block of 32 trials in which participants were exposed to all possible task transitions randomly. In the main experiment, there were eight blocks of 32 trials. The letter and the digit in each pair were randomly selected from the letter and digit pools with the constraint that, within a trial, no elements of 
Table 2

Task Carryover and Intertrial Relations for Possible Comparisons Between the Foreknowledge and the No-Foreknowledge Conditions in Experiments 1 and 2

\begin{tabular}{|c|c|c|c|c|}
\hline \multirow[b]{2}{*}{ Task pair } & \multicolumn{2}{|c|}{ Experiment 1} & \multicolumn{2}{|c|}{ Experiment 2} \\
\hline & Task carryover & Intertrial transition & Task cartyover & Intertrial transition \\
\hline LL-LL & Carryover & Identical & Carryover & Identical \\
\hline DD-LL & No carryover & Different & - & - \\
\hline LD-LL & - & - & - & - \\
\hline DL-LL & - & - & Carryover & Different \\
\hline LL-LD & - & - & - & - \\
\hline DD-LD & - & - & No carryover & Different \\
\hline LD-LD & No carryover & Identical & No carryover & Identical \\
\hline DL-LD & Carryover & Different & - & - \\
\hline
\end{tabular}

Note. A dash indicates that trial pairs were not possible in the foreknowledge conditions either in Experiment 1 or Experiment 2. $\mathrm{L}=$ letter; $\mathrm{D}=$ digit.

a Task 1 stimulus should be the same as those of a Task 2 stimulus. In the random condition, four types of sequences (i.e., LL, LD, DD, and DL) appeared eight times in a random order, resulting in 32 trials. In the foreknowledge condition, switch trials and repetition trials were blocked. Half of the participants in this condition performed task switches (LD and DL) in the first, third, fifth, and seventh blocks and task repetitions (LL and DD) in the second, fourth, sixth, and eighth blocks. The other half of the participants in this condition performed a different block-transition mapping. The resulting design was 2 (foreknowledge vs. no-foreknowledge transition) $\times 2$ (switch vs. repetition) mixed factorial.

Participants. Thirty-two college students recruited from an introductory psychology class at Pennsylvania State University participated in return for extra course credit. Sixteen participants were randomly assigned to each of the foreknowledge and no-foreknowledge conditions.

\section{Results and Discussion}

In the foreknowledge condition, practicing both types of transitions required two blocks. Therefore, in both conditions, the first two blocks were regarded as practice and were excluded from the analysis. The main analyses were two-way analyses of variance (ANOVAs), with foreknowledge condition (foreknowledge and no-foreknowledge) and task transition (repetition and switch) as factors for both accuracy and Task 2 latency. Task 1 and Task 2 latencies were also subjected to another three-way ANOVA to examine intertrial effects. Foreknowledge was a betweenparticipants factor, and task transition was a within-participants factor. In all experiments reported here, only correct trials were included in analyses of latency.

Accuracy. In all experiments, a trial was counted as correct only when both tasks in the trial were correct. The overall accuracy was .92. Accuracy was higher when the trial involved a task repetition (.94) than a task switch $(.91), F(1,30)=15.31, p<$ $.001, M S E=.001$. No other main effect or interaction was significant $(p>.50)$.

Task 2 latency. Overall latency was $940 \mathrm{~ms}$. Figure 2 shows the mean Task 2 latency as a function of task transition in each foreknowledge condition. Participants with foreknowledge (829 $\mathrm{ms})$ responded faster than those with no foreknowledge $(1,051$ $\mathrm{ms}), F(1,30)=10.58, p<.01, M S E=74642.28$. Participants took longer to perform Task 2 when it was switched from Task 1 $(1,058 \mathrm{~ms})$ than when it was repeated $(823 \mathrm{~ms}), F(1,30)=71.83$, $p<.0001, M S E=12334.11$. However, the interaction between task transition and the foreknowledge condition was not significant $(p>.40)$. Thus, foreknowledge and task transition appear to have additive effects on Task 2 latency. The fact that the switch cost is similar regardless of foreknowledge supports the idea that the source of switch cost is persisting activation from a previous task set, which changes automatically with no direct control from executive mechanisms. This interpretation is, of course, based on a null interaction. We test this hypothesis again in Experiment 2.

Intertrial effects. We examined higher order intertrial effects on Task 1 and Task 2 latencies. For Task 1, the question is whether there is carryover from Task 2 of the previous trial. Task 1 latency was subjected to a three-way ANOVA with foreknowledge, transition type of the previous trial (repetition or switch), and carryover relation between the previous Task 2 and the current Task 1 (carryover or no-carryover) as variables. The first trial of each block was excluded.

Figure 3 shows Task 1 latency plotted as a function of these variables (also refer to Table 2). Task 1 latency was faster when the previous trial involved repetition $(1,168 \mathrm{~ms})$ than switch $(1,353$ $\mathrm{ms}), F(1,30)=91.68, p<.0001, M S E=11893.89$. Task 1 latency was also faster with carryover from the previous Task 2 to the current Task $1(1,229 \mathrm{~ms})$ than with no-carryover $(1,292 \mathrm{~ms})$, $F(1,30)=20.35, p<.01, M S E=6198.15$. More importantly, these two variables interacted significantly, $F(1,30)=10.78, p<$ $.01, M S E=8187.60$. With a previous repetition, Task 1 was faster with carryover $(1,111 \mathrm{~ms})$ than with no carryover $(1,226 \mathrm{~ms})$, $t(31)=5.21, p=.0001$. For example, performing the underlined task in LL-L or DD-D sequence was faster than performing the same task in DD- $\underline{L}$ or LL- $\underline{D}$ sequence. However, this difference was not significant when the previous transition was switch $(p>$ .60 ). For example, performing the underlined task in LD-D or DL- $\underline{L}$ sequence was not different compared with the same task in LD- $\underline{L}$ or DL- $D$ sequence. This interaction indicates that, although there is an intertrial effect on Task 1, this does not depend only on the sequence of tasks. More important, this interaction did not depend on foreknowledge, suggesting that foreknowledge about Task 2 may not affect Task 1 performance.

For Task 2, intertrial effects might provide an alternative explanation for the effects discussed earlier. In the repetition block of 


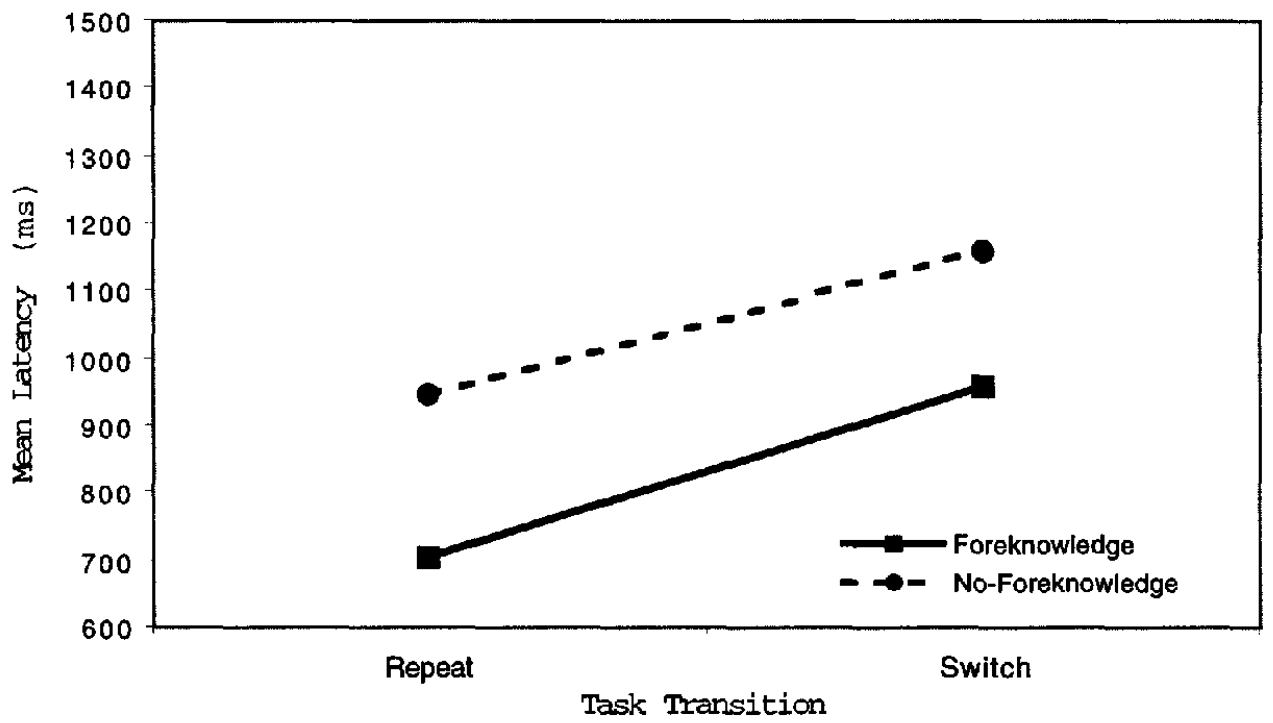

Figure 2. Mean Task 2 latency as a function of task transition in each foreknowledge condition in Experiment 1.

the foreknowledge condition, because all trials involved task repetition, there were only two types of intertrial transitions, as shown in Table 2, and the previous trial was always a repetition. Similarly, in the switch block of the foreknowledge condition, the previous trial was always a switch. However, in the noforeknowledge condition, all possible intertrial relations appeared. Because either identical-repetition or identical-switch cases could benefit performance, this factor was confounded with foreknowledge. To examine this possibility, a three-way ANOVA was performed with foreknowledge (foreknowledge vs. no foreknowledge), intertrial transition (identical vs. different), and within trial transition (repetition vs. switch). From the no-foreknowledge con- dition, we included only those trial pairs that matched trial pairs from the foreknowledge conditions. Because this analysis involves intertrial transitions, the first trials of the blocks were not included.

Figure 4 shows Task 2 latency plotted as a function of foreknowledge, intertrial transition relation, and within-trial transition. An explanation on the basis of the intertrial effect is that the benefit of the foreknowledge is due to the relatively greater proportion of identical intertrial transitions. If this is true, there should be no difference between foreknowledge and no-foreknowledge conditions, when comparing only those types of intertrial transitions from both conditions. However, performance with foreknowledge (825 ms) was still substantially faster than performance

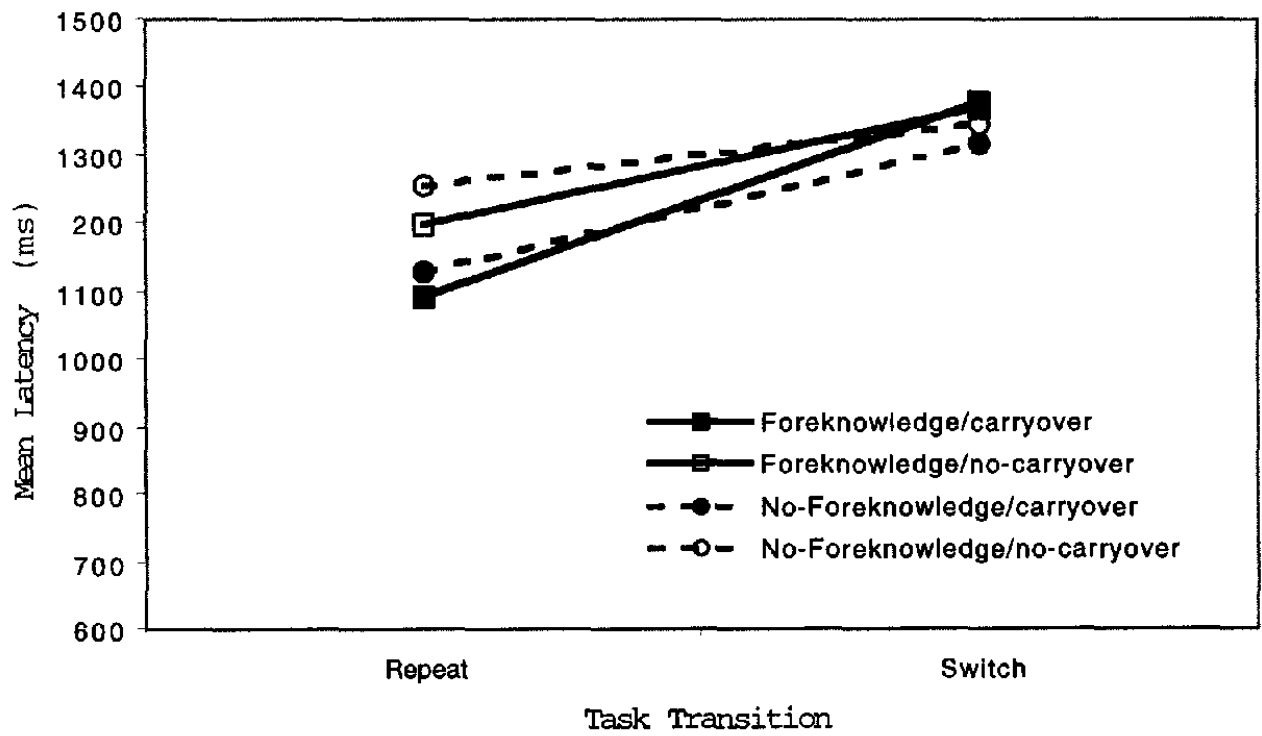

Figure 3. Mean Task 1 latency without the first trial in a block as a function of previous task transition and carryover in each foreknowledge condition in Experiment 1. 


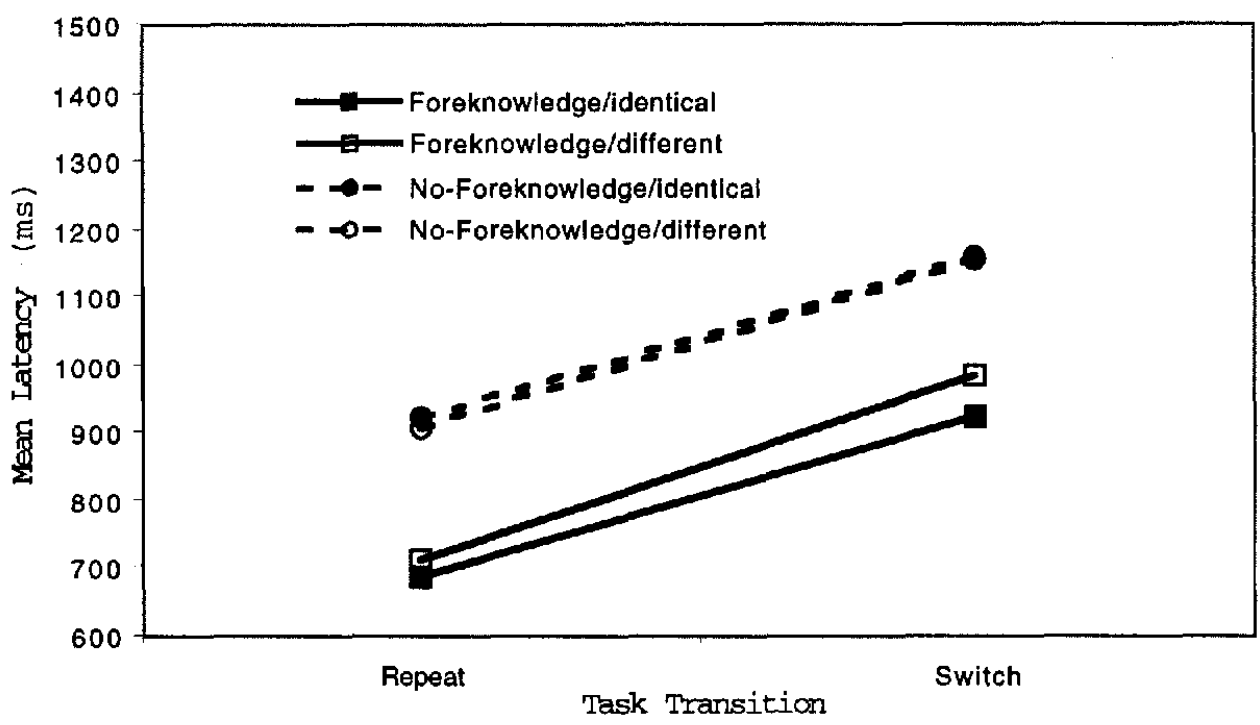

Figure 4. Mean Task 2 latency without the first trial in a block as a function of task transition and intertrial transition in each foreknowledge condition in Experiment 1.

with no foreknowledge $(1,035 \mathrm{~ms}), F(1,30)=9.96, p<.01$, $M S E=141830.61$. Performance with task repetition $(806 \mathrm{~ms})$ was still faster than performance with task switch $(1,054 \mathrm{~ms}), F(1$, 30) $=68.33, p<.0001, M S E=28754.18$. The interaction between foreknowledge and intertrial transition relation was marginally significant $(p<.09)$. In the foreknowledge condition, there was 41-ms benefit when two consecutive trials involved the identical transition (e.g., LL-LL or LD-LD) compared with when they did not (e.g., LL-DD or LD-DL), $t(15)=3.21, p<.01$. There was no such benefit in the no-foreknowledge condition. No other main effects or interactions were significant $(p>.3)$. Although repeating the same transition had greater impact in the foreknowledge condition than in the no-foreknowledge condition, this effect did not interact with transition type.

This intertrial analysis thus shows that carryover from a previous trial to the current trial cannot explain the pattern of switch costs. There are indeed intertrial effects, but they are not in the way to compromise the effects of foreknowledge or repetition. For Task 1, pattern of latency is not completely explained by intertrial effect. Moreover, Task 2 performance of the identical intertrial transition was not different depending on foreknowledge, and the amount of switch cost was not affected by the intertrial transitions. Therefore, the within-trial transition effect and foreknowledge effect on Task 2 latency reported earlier are not explained by higher order intertrial transition effects.

Congruence effect. In all experiments, Task 2 latencies were analyzed to examine whether a participant responded faster to a target when a response associated with the distractor was congruent with the response associated with the target. The ANOVA model to analyze the congruence effect was 2 (foreknowledge vs. no foreknowledge) $\times 2$ (congruent vs. incongruent) $\times 2$ (repetition vs. switch) mixed factorial. There were no significant main effect or interactions involving congruence in Experiment 1 (all ps $>$.1)

\section{Experiment 2}

The purpose of Experiment 2 was to replicate the results of Experiment 1 with a different foreknowledge manipulation. In Experiment 1, participants performed only task repetitions or only task switches within a block when there was foreknowledge, which is different from some of the previous studies. For example, in the Rogers and Monsell (1995) study, both task repetitions and task switches occurred in each block but in a predictable manner. This difference raises a question regarding whether participants may have adopted different strategies when task transitions were blocked. According to Strayer and Kramer (1994), when different experimental conditions were blocked, participants tended to adopt different strategies. However, when the conditions were mixed in one block, they tended to adopt one general strategy, although it may not have been an optimal strategy for all conditions. Los (1996) also suggested that mixing different types of trials may cause differences in strategies as well as differential adaptation to trail-by-trial changes.

To examine whether the results in Experiment 1 were an artifact of blocking task transitions, task identity rather than task transition was blocked or random in Experiment 2. Table 3 illustrates the foreknowledge manipulations in Experiment 2. Participants in the foreknowledge condition received two different types of blocks, and they were explicitly instructed about the different organization of each block. In the letter block, participants were instructed that, although Task 1 would be either the letter task or the digit task, Task 2 would always be the letter task. In the digit block, participants were instructed that, although Task 1 would be either the letter task or the digit task, Task 2 would always be the digit task. Therefore, in this condition, unlike in the foreknowledge condition of Experiment 1, task repetitions and task switches were randomly mixed, but foreknowledge about Task 2 was always available. In contrast, for participants in the no-foreknowledge condition, Task 1 and Task 2 were the letter task or the digit task equally 
Table 3

An Illustration of the Foreknowledge Manipulations in Experiment 2

\begin{tabular}{llll}
\hline \multicolumn{1}{c}{ Condition } & Type of block & Task 1 & Task 2 \\
\hline Foreknowledge & Letter block & Letter & Letter \\
& & Digit & Letter \\
& Digit block & Letter & Digit \\
& & Digit & Digit \\
No foreknowledge & Mixed block & Letter & Letter \\
& & Letter & Digit \\
& & Digit & Letter \\
& & Digit & Digit \\
\hline
\end{tabular}

often, the same as in the no-foreknowledge condition of Experiment 1.

Foreknowledge about both task transition and task identity was available in the foreknowledge condition as in Experiment 1 , and the no-foreknowledge condition was exactly the same as in Experiment 1 . Therefore, the same pattern of results was expected as in Experiment 1, if the results of Experiment 1 were not due to blocking of task transitions.

\section{Method}

Task and equipment. Stimuli and tasks were the same as in Experiment 1.

Procedure and design. The procedure was the same as in Experiment 1, except that Task 2 was always the letter task or the digit task depending on a particular block in the foreknowledge condition. The resulting design was a 2 (foreknowledge vs. no foreknowledge) $\times 2$ (switch vs. repetition) mixed factorial.

Participants. Thirty-two college students recruited from an introductory psychology class at Pennsylvania State University participated in return for extra course credit. Sixteen participants were randomly assigned to each of the foreknowledge and no-foreknowledge conditions.

\section{Results and Discussion}

As in Experiment 1, the first two blocks were regarded as practice and were excluded from the analysis.

Accuracy. The overall accuracy was .90 . Accuracy was higher when the trial involved a task repetition (.92) than a task switch (.87), $F(1,30)=19.05, p<.001, M S E=.002$. No other main effect or interaction was significant $(p>10)$.

Task 2 latency. Overall latency was $993 \mathrm{~ms}$. Figure 5 shows the mean Task 2 latency as a function of task transition in each foreknowledge condition. Task 2 latency was faster with foreknowledge (912 ms) than with no foreknowledge (1,074 ms), $F(1$, $30)=4.62, p<.05, M S E=91062.09$. Task 2 latency was faster when Task 2 was repeated $(887 \mathrm{~ms})$ than when it was switched $(1,095 \mathrm{~ms}), F(1,30)=79.43, p<.0001, M S E=8993.41$. However, as in Experiment 1, the interaction between task transition and the foreknowledge condition was not significant $(p>$ $.50)$. These results are consistent with Experiment 1. The replicated foreknowledge advantage indicates that foreknowledge allowed advance initiation of preparation. The fact that both main effects of task transition and the foreknowledge condition were significant without interacting, as in Experiment 1, is consistent with the idea that the source of the switch cost may be persisting activation from the previous task.

Although these results are consistent between Experiments 1 and 2, the supporting evidence for the persisting activation as the source of the switch cost is based in part on null interactions between foreknowledge and task transition. Because this raises a concern about a possible lack of power, Task 2 results from both experiments were subjected to a further test. The ANOVA model was 2 (Experiment 1 vs. 2) $\times 2$ (foreknowledge vs. no foreknowledge) $\times 2$ (task repetition vs. task switch) mixed factorial. Consistent with earlier analyses, only the main effects of foreknowledge and task transition were significant, $F(1,60)=14.26, p<$ $.01, M S E=82852.18$, and $F(1,60)=149.64, p<.0001, M S E=$

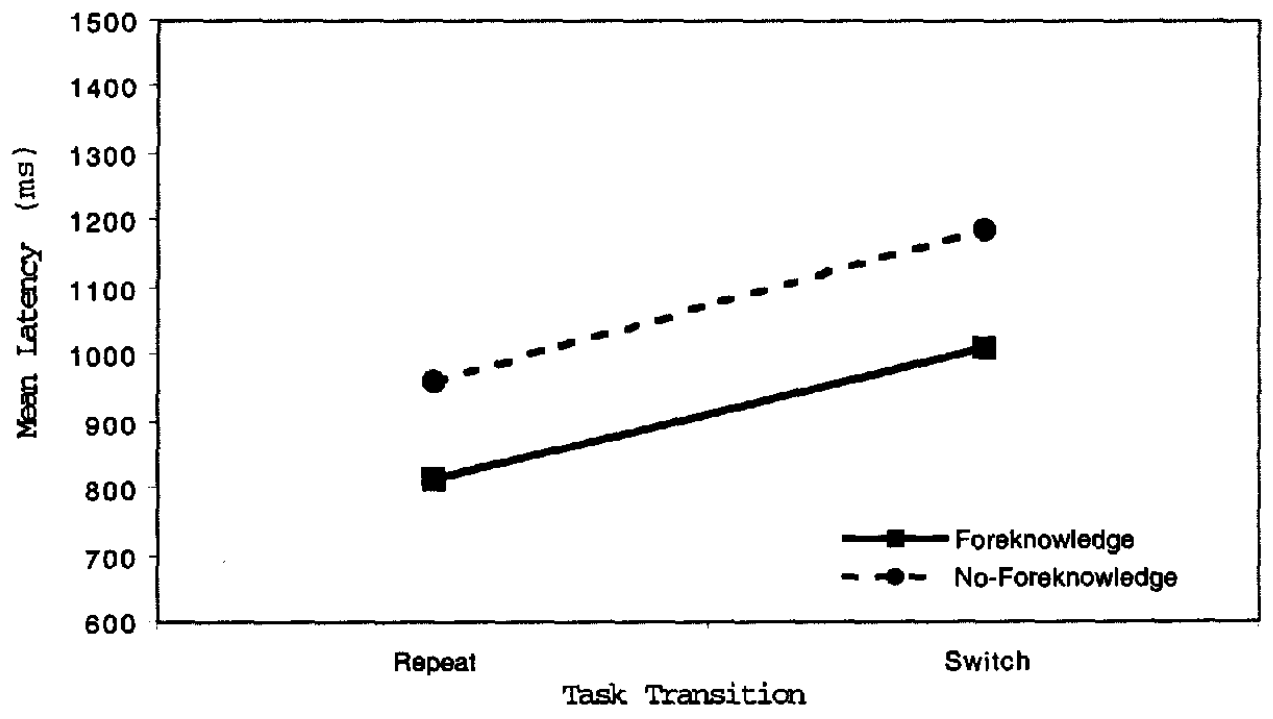

Figure 5. Mean Task 2 latency as a function of task transition in each foreknowledge condition in Experiment 2. 
10663.76. No other main effect and interactions were significant $(p>.30)$. This analysis suggests that the null interactions found in Experiments 1 and 2 with Task 2 latency were not due to lack of power.

Intertrial effects. As in Experiment 1, we examined intertrial effects. For Task 1, the same three-way ANOVA model was used. Figure 6 shows Task 1 latency plotted as a function of foreknowledge, previous transition, and carryover. Task 1 latency was faster after repetition trials $(1,202 \mathrm{~ms})$ than after previous switch trials $(1,385 \mathrm{~ms}), F(1,30)=105.85, p<.0001, M S E=10074.39$, and faster with carryover $(1,250 \mathrm{~ms})$ than with no carryover $(1,338$ $\mathrm{ms}), F(1,30)=24.47, p<.01, M S E=10046.13$. These two variables interacted significantly, $F(1,30)=11.47, p<.01$, $M S E=8524.08$. The pattern of interaction is consistent with Experiment 1. After repetition trials, Task 1 was faster with carryover $(1,131 \mathrm{~ms})$ than with no carryover $(1,274 \mathrm{~ms})$, $t(31)=6.20, p=.0001$. For example, performing the underlined task in $\mathrm{LL}-\mathrm{L}$ or $\mathrm{DD}-\mathrm{D}$ sequence was faster than performing the same task in DD- $\underline{L}$ or LL- $\underline{D}$ sequence. However, this difference was not significant when following switch trials $(p>.10)$. For example, performing the underlined task in LD- $\underline{D}$ or DL-L sequence was not different compared with the same task in LD- $\underline{\mathrm{L}}$ or DL-D sequence. The pattern of results is exactly the same as in Experiment 1.

Because task identity rather than task transition was blocked in the foreknowledge condition of this experiment, the analysis of Task 2 involved a different selection of trial pairs from the foreknowledge condition (see Table 2). We examined intertrial effects for Task 2 latency using a three-way mixed ANOVA model with foreknowledge (foreknowledge or no foreknowledge), intertrial transition (identical or different), and within-trial transition (repetition or switch) as factors.

Figure 7 shows Task 2 latency plotted as a function of foreknowledge, intertrial transition relation, and within-trial transition. An explanation on the basis of intertrial effects would be that the benefit of foreknowledge is due to the relatively greater proportion of identical intertrial transitions. If this is true, there should be no difference between foreknowledge and no-foreknowledge conditions when comparing only the same types of intertrial transitions from both conditions. However, performance with foreknowledge (903 ms) was still faster than performance with no foreknowledge $(1,075 \mathrm{~ms}), F(1,30)=4.99, p<.05, M S E=188294.06$. Performance with task repetition $(878 \mathrm{~ms})$ was still faster than performance with task switch $(1,100 \mathrm{~ms}), F(1,30)=82.50, p<.0001$, $M S E=19012.94$. The main effect of intertrial transition was significant, $F(1,30)=6.48, p<.05, M S E=6940.34$. Performance was faster when the intertrial transition was identical (970 ms) than when different $(1,008 \mathrm{~ms})$. The interaction between within-trial transition and intertrial transition was significant, $F(1$, $30)=15.61, p<.01, M S E=6760.65$. As shown in Figure 7 , performing a task repetition after performing a repetition on the previous trial (e.g., LL-LL or DD-DD, $831 \mathrm{~ms}$ ) was faster than performing a task repetition after performing a switch on the previous trial (e.g., LD-DD or DL-LL, $926 \mathrm{~ms}$ ), $t(31)=6.37, p<$ .0001 . However, there was no such difference when the current transition was a switch $(p>.40)$.

Consistent with Experiment 1, the intertrial analysis of Experiment 2 also suggests that, although there are intertrial effects, they do not provide an alternative explanation for the effects of foreknowledge or repetition. Task 1 latency is not completely under the influence of higher order intertrial effects. In Task 2 latency, the intertrial effects do not explain the switch cost. Therefore, interpretation of Task 2 latency in terms of foreknowledge and within-trial transition effects does not seem to be compromised by intertrial effects.

Congruence effect. In this experiment, the interaction between congruence and task transition in Task 2 latency was significant, $F(1,30)=5.56, p<.05, M S E=3523.84$. When the transition was a repetition, Task 2 latency was slower with a distractor associated with the opposite response ( $894 \mathrm{~ms}$ ) than with a dis-

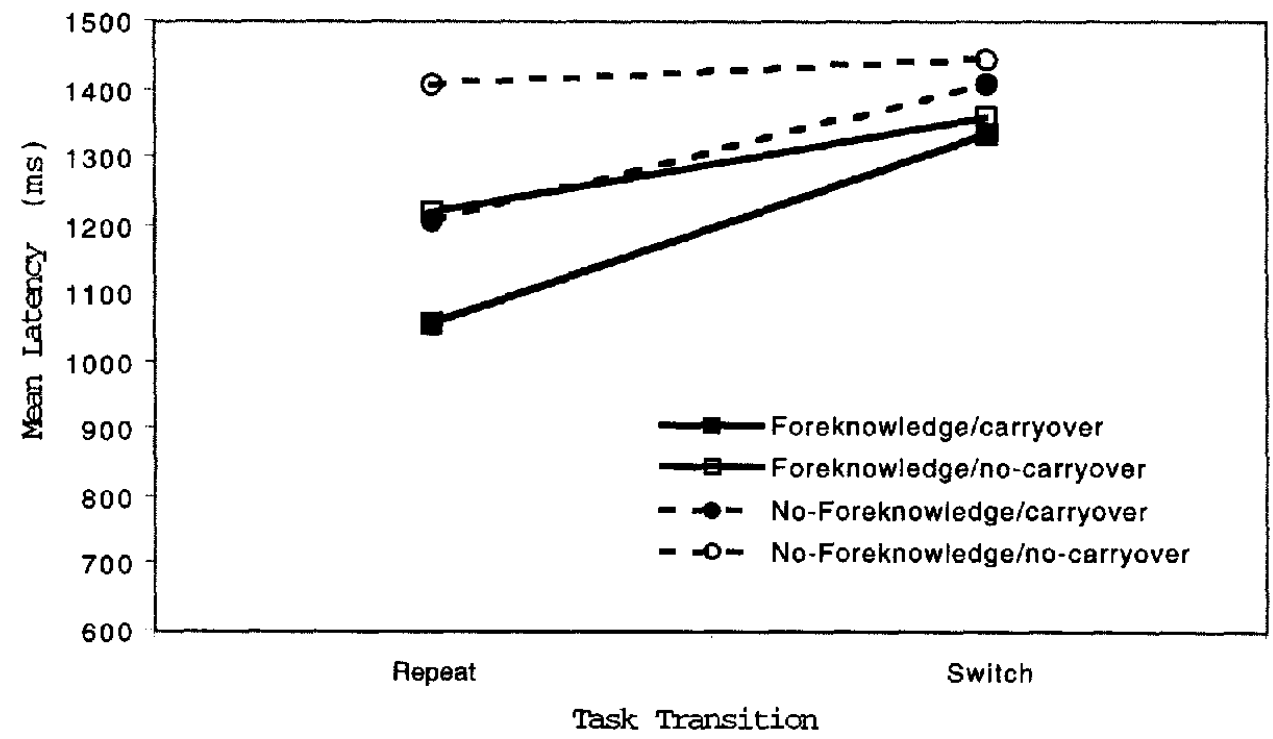

Figure 6. Mean Task 1 latency without the first trial in a block as a function of previous task transition and carryover in each foreknowledge condition in Experiment 2. 


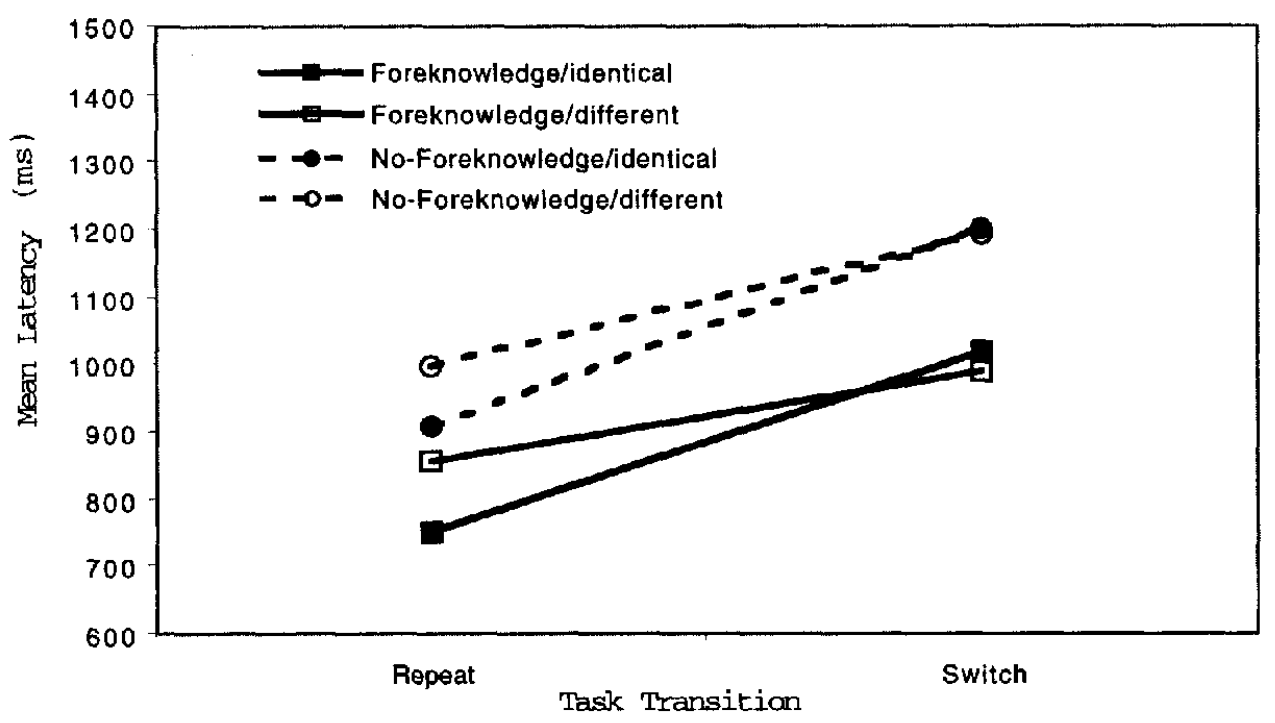

Figure 7. Mean Task 2 latency without the first trial in a block as a function of task transition and intertrial transition in each foreknowledge condition in Experiment 2.

tractor associated with the congruent response $(880 \mathrm{~ms})$, but this difference was not significant $(p>.30)$. When the transition was a switch, Task 2 latency was faster with a distractor associated with the opposite response $(1,080 \mathrm{~ms})$ than with a distractor associated with the congruent response $(1,115 \mathrm{~ms})$, and this difference was only marginally significant, $t(32)=2.03, p<.06$. Although the significant interaction between congruence and task transition seems to be due to opposite congruence effects in each task transition, the size of these nonsignificant simple effects was quite small relative to the switch cost obtained in this experiment. In general, the lack of interaction between congruence and the foreknowledge condition indicates that, although congruence may affect response execution, it does not affect the preparation process of task-set reconfiguration.

\section{Experiment 3}

The results of Experiments 1 and 2 suggest that the switch cost can be attributed to automatic activation from Task 1 that does not disappear completely rather than to inadequate preparation for a new task. With task repetitions, this persisting activation may result in facilitation by repetition priming. With task switches, the same persisting activation may interfere with the current task. This interpretation is based on the lack of interaction between foreknowledge and task transition. The main effect of foreknowledge indicates that participants could prepare for both repetitions and switches on the basis of foreknowledge during the RSI before Task 2 stimulus presentation. However, because only two task sets were used in these experiments, it is not clear whether this preparation was based only on task transition information or on information about a specific task set.

The hypothesis we are testing in the current study is that the effect of foreknowledge is independent of the effect of repetition. Our assumption was that the extent of preparation is the same for both repetition and switch as long as the same amount of infor- mation is available. For example, in the foreknowledge conditions in previous experiments, foreknowledge about both task transition and task identity was available, whereas neither piece of information was available in the no-foreknowledge conditions. We argue that, in each condition, the extent to which a task is prepared should be the same regardless of task transition. This assumption is not entirely consistent with several studies arguing that the nature of the switch cost is inadequate preparation for a switched task set compared to repeated task set (De Jong, in press; De Jong et al., 1999; Rogers \& Monsell, 1995). Although to our knowledge it has not been discussed in the literature, one form of less-thanperfect preparation for a task switch might be preparation that is not specific to a task set. That is, with foreknowledge about task transition, participants may prepare themselves for a task switch but not specifically for the upcoming task set. In contrast, when the transition is repetition, because the task has been performed already, participants may be able to specifically prepare for the repeated task set. In Experiment 3, we tested whether the preparation for a task switch is on the basis of a specific task set to be adopted or simply on the basis of the knowledge that a switch would occur.

Experiment 3 used three tasks to dissociate task-transition and task-identity information. Transition type was always blocked, so that all participants had global foreknowledge of transition type. However, for half of participants, foreknowledge specified both task transition and task identity: the full-foreknowledge condition. For the other half of participants, foreknowledge specified only task transition information: the partial-foreknowledge condition. Table 4 illustrates the foreknowledge manipulations in Experiment 3 . Notice that repetition condition is exactly the same in both full- and partial-foreknowledge conditions. The difference between the conditions lies in the amount of information conveyed by foreknowledge regarding a task switch. Because the repetition performance should be the same in both conditions, this manipulation allows an interesting inference about the nature of the 
Table 4

An Illustration of the Contingency Manipulations in Experiment 3

\begin{tabular}{clll}
\hline \multicolumn{1}{c}{ Condition } & Type of block & Task 1 & Task 2 \\
\hline Full foreknowledge & Repetition block & Letter & Letter \\
& & Digit & Digit \\
& Switch block & Symbol & Symbol \\
& & Letter & Digit \\
& & Digit & Symbol \\
& & Symbol & Letter \\
Partial foreknowledge & Repetition block & Letter & Letter \\
& & Digit & Digit \\
& & Symbol & Symbol \\
& Switch block & Letter & Digit \\
& & Letter & Symbol \\
& & Digit & Letter \\
& & Digit & Symbol \\
& & Symbol & Letter \\
& & Symbol & Digit \\
\hline & & & \\
& & &
\end{tabular}

preparation for Task 2 during RSI. If preparation for a task switch is specific for a task set, this should be possible only with full foreknowledge. That is, if Task 2 is, in fact, prepared while activation of Task 1 diminishes, the switch cost with partial foreknowledge should be greater than with full foreknowledge. However, if preparation for a task switch is on the basis of knowledge only of an impending task switch, there should be no difference between full and partial foreknowledge. Because of the different structure of the design for this experiment, we did not analyze intertrial effects.

\section{Method}

Task and equipment. Stimuli and tasks were the same as in Experiments $I$ and 2 with one additional task and corresponding element in each stimulus. The additional task was the symbol task, which was to decide whether a typographic symbol had curved lines (@, \$, \%, and \&) or only straight lines $\left(!, \#,{ }^{\wedge}\right.$, and *). The stimulus consisted of three characters: one letter, one digit, and one symbol in a random order. Among these three, one was a target, and the other two were distractors. Distractors were selected so that one of them would be associated with the same response as the target, and the other would be associated with the opposite response as the target. Therefore, congruence between the responses associated with the target and distractors is not an issue in this experiment. Because there were three task sets, an additional color, blue (MEL color code 1), was used to indicate a task set.

Procedure and design. The procedure was the same as the foreknowledge condition in Experiment 1 with the addition of the symbol task described earlier. Consequently, one response key was mapped to three responses. The "even," "consonant," and "straight" responses were assigned to one key, and the "odd," "vowel," and "curved" responses were assigned to another key. At the beginning of each experimental session, there was a practice block of $\mathbf{4 8}$ trials in which participants were exposed to all possible task transitions randomly. In the main experiment, there were six blocks of 48 trials. For half of the participants, Task 2 was contingent on Task 1 in switch blocks, full-foreknowledge condition. For the other half of the participants, Task 2 was not contingent on Task 1 in switch blocks, partial-foreknowledge condition. The resulting design was a 2 (full vs. partial foreknowledge) $\times 2$ (switch vs. repetition) mixed factorial.

Participants. Thirty-two college students recruited from an introductory psychology class at Pennsylvania State University participated in return for extra course credit. Sixteen participants were randomly assigned to each of the full-foreknowledge and partial-foreknowledge conditions.

\section{Results and Discussion}

In both foreknowledge conditions, the first two blocks were regarded as practice and were excluded from the analysis.

Accuracy. The overall accuracy was $\mathbf{9 2}$. Accuracy was higher when the trial was a repetition (.93) than a switch (.91), $F(1$, $30)=5.87, p<.001, M S E=.05$. Accuracy was higher with full foreknowledge about Task 2 task identity as well as task transition (.94) than with partial foreknowledge about task transition (.91), $F(1,30)=4.24, p<.05, M S E=.005$. The interaction was not significant $(p>.70)$.

Task 2 latency. Overall mean latency was 896 ms. Figure 8 shows the mean Task 2 latency as a function of task transition in each foreknowledge condition. Task 2 latency was faster when a Task 2 was repeated $(690 \mathrm{~ms})$ than when it was switched $(1,102$ $\mathrm{ms}), F(1,30)=158.86, p<.0001, M S E=17161.80$. The main effect of foreknowledge was not significant $(p>1)$. However, this switch cost depended on foreknowledge, $F(1,30)=9.42, p<$ $.01, M S E=17161.80$. Task 2 switch cost was smaller when there was foreknowledge about both task transition and task identity, as in the full-foreknowledge condition, than when there was foreknowledge about only task transition, as in the partialforeknowledge condition. This result can be explained by assuming that a switched task in the full-foreknowledge condition was prepared while the priming effect from Task 1 was also operating. In contrast, for a task switch in the partial foreknowledge condition, preparation for a specific task set was not possible. Recall that the amount of information conveyed for task repetition was the same regardless of foreknowledge condition. Knowing the current task will be repeated (task transition foreknowledge) naturally conveys the task identity information also. In fact, means of task repetition latencies from both conditions were nearly identical. Therefore, it seems that not only switches but also repetitions take advantage of foreknowledge for advance preparation. The results of Experiment 3 show that foreknowledge provided in previous experiments could allow preparation for a specific new task set, while priming from Task 1 is still operating.

\section{General Discussion}

The current results and their implications can be summarized as follows: First, a task switch cost was consistently found in all experiments in all conditions. The switch cost did not differ for full-foreknowledge and no-foreknowledge conditions (Experiments 1 and 2). This result suggests that the source of switch cost is persisting activation from the previous task, which provides a repetition benefit for a repeated task but interference for a switched task. However, the switch cost was greater with only partial foreknowledge about task transition than with full foreknowledge about both task transition and task identity (Experiment 3). This result suggests that knowledge about a specific task set is used to prepare for a switched task while the persisting activation from a previous task decreases.

Second, foreknowledge facilitated performance. Full foreknowledge of both task transition and task identity always speeded Task 2 responses compared with no foreknowledge (Experj- 


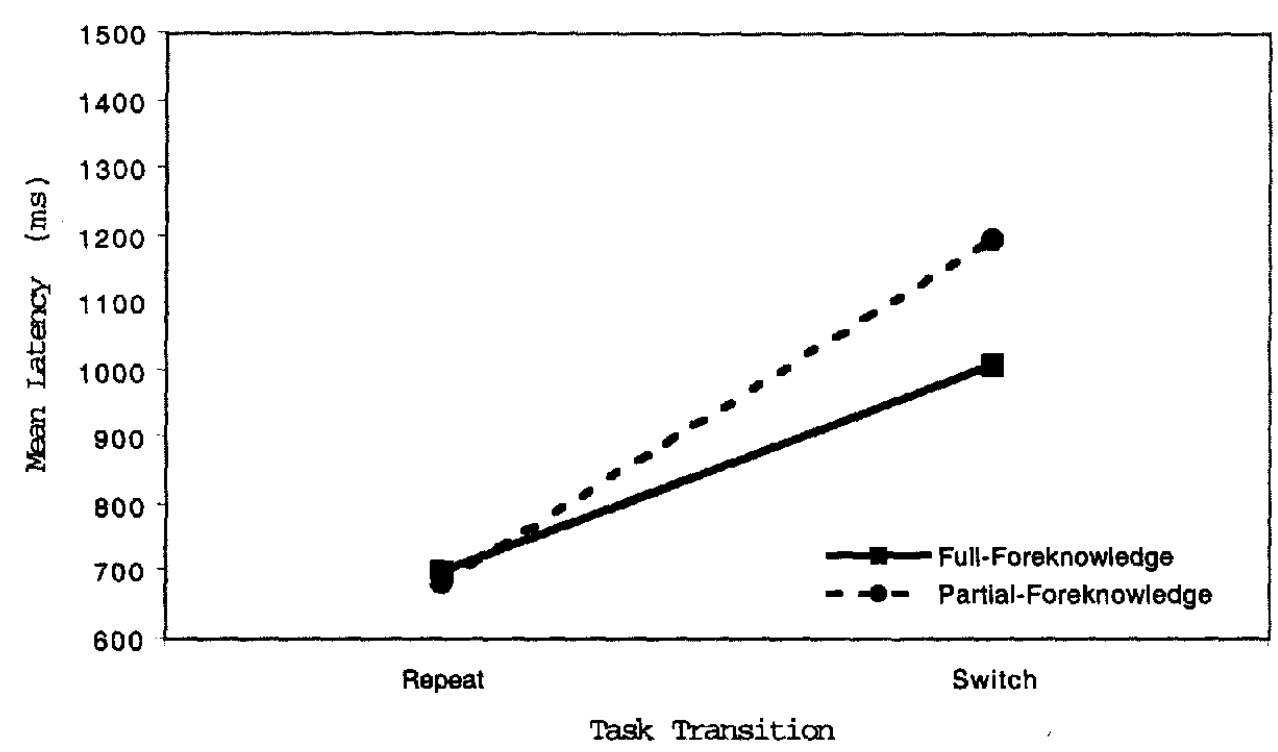

Figure 8. Mean Task 2 latency as a function of task transition in each contingency condition in Experiment 3.

ments 1 and 2) and partial foreknowledge of just task transition (Experiment 3). Moreover, foreknowledge facilitated both repetitions and switches. These results suggest that task identity foreknowledge allowed participants to initiate preparation for Task 2 before the stimulus presentation, regardless of whether the task is repeated or not.

Third, we found intertrial effects for both Task 1 and Task 2 latencies. With a previous trial involving task repetition, Task 1 latency was faster when it was carried over from the previous trial than when it was not. However, this carryover did not have any effect when the previous trial involved a task switch. Task 2 latency was faster when the current trial was the exact copy of the previous trial. However, this intertrial transition effect did not affect the switch cost or depend on foreknowledge. Therefore, although there is some effect from intertrial transition, this does not seem to undermine our interpretation of the source of the switch cost or the effects of foreknowledge.

Altogether, the current results present a picture that is rather inconsistent with the view that the source of the switch cost is inadequate preparation for the switched task set. If the switch cost reflects inadequate preparation, then the amount of switch cost should depend on foreknowledge availability. This is because the extent of preparation may depend on task transition with foreknowledge but may not be different with no foreknowledge. Instead, the current results are consistent with the view that switch cost reflects something about the task that has just been performed. That is, once a task is performed, its activation persists for some time, facilitating the performance of the same task but interfering with the performance of a different task.

\section{Preparation}

The current experimental manipulations have some methodological advantages over previous studies. As noted earlier, many previous task-switching studies adopted rather a long sequence of steps with predictable task transitions, which makes it difficult to dissociate preparation from performance. In the current paradigm, by shortening the length of a sequence and focusing on the last task of the sequence, it was possible to examine the effect of task transition while minimizing the influence of sequential effects. Also, the number of relevant task sets in a block was always the same across different conditions, as opposed to the shift paradigm (e.g., Allport et al., 1994). Although sometimes only task repetitions or task switches were performed within a block (Experiments 1 and 3), the results did not differ qualitatively when both types of transitions were randomly mixed (Experiment 2).

Rogers and Monsell (1995) manipulated RSIs and found that the switch cost did not decrease further after about 600 -ms RSI. Because this residual switch cost seemed to be limited to the first step after a switch (Gopher et al., 1998; Rogers \& Monsell, 1995), it has been attributed to inadequate preparation (Rogers \& Monsell, 1995) or occasional failure of preparation (De Jong, in press; De Jong et al., 1999). The point is that the very first performance of a task is special, and its engagement requires an external stimulus (Rogers \& Monsell, 1995). However, because these studies allowed foreknowledge about transition sequence in one way or another, it is not entirely clear whether the incomplete process is preparation for a new task or a reduction of persisting activation from a previous task. Moreover, if we assume that reduction of persisting activation can also be completed on the basis of the first stimulus for a new task (Gopher et al., 1998), there is no reason to assume further that the interference on the basis of the persisting activation should last for a few steps after the switch. If an external stimulus is either necessary or sufficient to complete either preparation or reduction of persisting activation, manipulating RSI alone may not be adequate to observe the time course of the processes involved in task switching.

Meiran (1996) manipulated cue-to-target interval rather than RSI and found that the switch cost was reduced as the cue-to-target interval increased, even when RSI was held constant. He argued that the process operating before stimulus onset is "retroactive 
adjustment" to initiate the next task set rather than the previous task set. Because the switch cost did not disappear even with a long cue-to-target interval, Meiran argued that there are both reducible and irreducible switch costs. Moreover, because task transition was predictable in most studies with RSI manipulations, an RSI can also be regarded as a cue-to-target interval.

Gopher et al. (1998) reported results showing that reconsideration of the same task set was always faster than switching to a different task set, suggesting that the residual switch cost may include the cost related to clearing the system of the old task set for a new task set. In their studies, at a randomly chosen step among 15, a participant either reconsidered the previous task or considered a new task. Even when both repetitions and switches were explicitly cued and there was a substantial amount of time $(1,200 \mathrm{~ms})$ to prepare, it took more time to perform a switch than a repetition. On the assumption that endogenous preparation for both repetitions and switches must have been equal, Gopher et al. attributed this switch cost to a stimulus-related, bottom-up process. However, once again because they did not manipulate foreknowledge or number of tasks, it is not entirely clear whether this process is to finish inadequate preparation for a switched task or to reduce the lingering activation from the previous task.

The current study adopted foreknowledge rather than RSI manipulations because foreknowledge may have different consequences for preparation and persisting activation. The benefit of repetition may exert its effects regardless of foreknowledge. However, preparation for a new task set requires knowledge of the new task identity. If the switch cost reflects simply persisting activation, the amount of switch cost should not depend on foreknowledge. In contrast, if the switch cost reflects inadequate preparation, the amount of switch cost should be greater with foreknowledge about task identity. This is because, with no foreknowledge, the extent of preparation for repetition and switch should be equivalent. Therefore, Task 2 latency in this condition will include the entire cost of preparation for both repetition and switch trials. However, the switch cost was no different when compared between full foreknowledge and no foreknowledge at all, supporting the idea that switch cost may be due to persisting activation. Also in the current study, task-transition information was dissociated from task-identity information by using more than two task sets. Foreknowledge about only task transitions resulted in greater switch cost compared with foreknowledge about both task transition and task identity. This result confirms that preparation even for a switched task set could be endogenously initiated when there was foreknowledge about task identity.

\section{Persisting Activation}

A critical assumption of the current study is that the extent of preparation for switch and repetition is the same: prepared with foreknowledge or not prepared without foreknowledge. If so, any difference between task switch and task repetition in noforeknowledge condition should be attributed to persisting activation. Consequently, if the amount of switch cost in the foreknowledge condition is the same as that in the no-foreknowledge condition, that should also be attributed to persisting activation. It is important to make sure that the equal-preparation assumption is plausible, especially with no foreknowledge. Consider three alter- native strategies available to participants with no-foreknowledge on completion of Task 1 :

1. Actively maintain the current task set. This strategy should be beneficial on repetition trials and harmful on switch trials. If this strategy is adopted, there would be no advantage of foreknowledge on repetition trials and a large advantage on switch trials.

2. Guess which task set will be required on Task 2, and engage that task set. This will be beneficial when the guess is correct and harmful when it is not. In this case, there should be no (or at least reduced) average switch cost with no foreknowledge, because on average half of repetition trials will involve switching from the guessed task set, and half of switch trials will involve actually using the guessed task set.

3. Do not prepare any task sets and adopt a new task set or re-engage the previous task set on the basis of Task 2 stimulus. In this case, foreknowledge should speed Task 2 responses by approximately equal amounts for repetition and switch trials.

The results support Alternative 3. Furthermore, if the residual switch cost were due to the need to complete preparation exogenously, foreknowledge should speed Task 2 responses more on repetition trials than on switch trials. This is because preparation for the appropriate task set can be completed on the basis of Task 1 stimulus and maintained on the basis of foreknowledge that the task set will be used again on Task 2 . The overall pattern of results thus suggests that the extent of preparation (or no preparation) was the same for repetition and switch with no foreknowledge available.

The idea of persisting activation as the source of the residual switch cost is consistent with that of Allport et al. (1994). They argued that, to switch to a different task set, additional time is required to settle down to a unique response decision because there is inertia from the previous task set. According to Allport et al., this inertia completely disappears only on the basis of an external stimulus, interfering with the execution of a new task set. We argued that, although the activation of a just-performed task may start to diminish as soon as the task has been performed, its complete reduction requires an external stimulus that explicitly supports another task set. Why might this be so? One possibility is that the remaining activation is further reduced only by allocating it to a new task set on the basis of an external stimulus. In the current paradigm, this allocation process might have been hindered because of a distractor that reminds participants of the previous task set. If this is true, this process should be faster when the external stimulus carries no distractor associated with a previous task set, because the distractor may serve as an exogenous source of activation that supports a previous task set. Some data support this possibility; Ruthruff et al. (1996) used a stimulus consisting of only one element without a distractor. In their experiments, the switch cost was 50 to $60 \mathrm{~ms}$, which is relatively small compared with that of other studies using distractors. For example, in the current studies, the switch cost ranged from 200 to $500 \mathrm{~ms}$. Also, in Rogers and Monsell (1995), when distractors were neutral, in the sense that they were not associated with any task sets, the size of the switch cost was almost half of the switch cost when distractors were associated with either compatible or incompatible responses.

This hypothesis of activation decrease by replacement contrasts with the decrease of activation only as a function of time, as suggested by Allport et al. (1994). However, the current paradigm does not allow a test of whether this process would have gradual 
effects or not, because there were only two tasks within a sequence. As noted earlier, many studies rejected the concept of inertia by showing that residual switch cost is limited to the first step after a switch (Rogers \& Monsell, 1995; Gopher et al., 1998). Although Meiran (1996) found a gradual decline of switch cost, he interpreted this as the continuing adjustment of the cognitive system to a new task set rather than interference from a previous task set. However, none of these studies compared foreknowledge with no foreknowledge, as in the current studies. An interesting question is whether foreknowledge would affect the residual switch cost over several steps after the first switch.

\section{Relation to Models of Executive Control}

A one-sentence summary of the current study would be that the effect of persisting activation may be independent of the effect of foreknowledge. How does this relate to our initial distinction between executive control and automatic control? Perhaps executive control can initiate preparation of a new task on the basis of foreknowledge or whatever kind of information that is available at the moment. Automatic control may be responsible for the effects of persisting activation, whenever the previous task is repeated. In fact, this line of logic is not new at all. It has been a common implication of previous studies on executive-automatic control that activation change from automatic priming is not under the control of executive mechanism (Neely, 1977; Schneider \& Shiffrin, 1977; Posner \& Snyder, 1975). For example, Neely (1977) showed that time course of automatic priming may be independent of whether or not participants could anticipate the next stimulus. Adding RSI manipulation to a design similar to the current study, Sohn and Anderson (1999) also replicated the independence of foreknowledge and task transition with relatively long RSIs. In this section, we explore how our notion of independence of repetition and foreknowledge may fit in existing models of cognitive control.

Logan (1985) suggested that executive control includes engagement or choice of a task set (strategy in his terminology), construction and maintenance of the task set, and disengagement or disablement of the task set. Among these, engagement and disengagement are of particular interest in the current study. Engagement of a task set is choosing among several task sets, each of which relates a response to target, the relevant aspect of a stimulus. The fact that executive control engages a task set may be consistent with our notion that preparation for a task is controlled by executive mechanism. When the goal of the current task is achieved and no longer necessary, the task set should be disabled so that other task sets can be engaged; this process is disengagement.

Because disengagement may be equivalent to reduction of activation, Logan's implication may be that even reduction of activation is initiated by an executive mechanism. It has been argued that executive functions also include monitoring the level of activation of different task sets (e.g., Norman \& Shallice, 1980; Shallice, 1994) and whether the current goal is achieved or not (Anderson, 1983, 1993) and scheduling execution of different task sets when more than one task should be performed within a limited time (Meyer \& Kieras, 1997). The scheduling may involve executive decisions about when to initiate engagement or disengagement of a task set. The timing can be critical because many theories assume that cognitive processing ability is limited as a result of either a limited-capacity central processing mechanism
(Baddeley, 1986; Norman \& Shallice, 1980) or limits in peripheral sensory-motor systems (Meyer \& Kieras, 1997). For example, activation might be distributed over different task sets so that multiple task sets may be activated to some degree, but the total amount of activation available at one time may be limited. By issuing signals to change the level of activation at appropriate times, an executive mechanism may allocate this total amount of activation appropriately. The current data cannot confirm nor deny this possibility. However, it is certainly an interesting area to pursue further for future study.

The possibility of active control over persisting activation was also suggested by Ruthruff et al. (1996). Using Rogers and Monsell's (1995) paradigm, Ruthruff et al. manipulated the extent of task set predictability so that a certain task set should be highly but not completely predictable (i.e., .8 probability). Therefore, some repetition and switch are expected but some are unexpected. They reasoned that if the switch cost results from automatic control, this cost should not depend on whether the repetition was expected or not. However, the switch cost was smaller when repetition was unexpected. When they compared the cost between a completely predictable case (1.0) and a completely random case (.5), which is similar to our Experiments 1 and 2, the switch cost was comparable. Although it would be interesting to pursue further how much control the executive mechanism has over the switch cost, it is questionable whether probability manipulation would be the only way to go. Suppose that the current Task $\mathrm{L}$ is biased to be switched to Task D with .8 expectancy, but in fact Task L is unexpectedly repeated. In this case, even if there was automatic priming from repetition, this effect might be washed out by the process in which the prepared Task $\mathrm{D}$ is canceled and Task $\mathrm{L}$ is reloaded. Therefore, it is possible that the lack of a persisting activation effect may, in fact, reflect the cost of canceling the current intention.

According to Norman and Shallice (1980; Shallice, 1994), executive control is achieved by a supervisory attentional system (SAS). They argued that activation of a task set strongly associated with a salient aspect of an external stimulus is exogenously increased to be selected for execution. However, because more than one action or thought schema may be available at a given time, sometimes a dominant schema may not emerge. Task switching requires planning because execution of a new task should be scheduled in relation to the current task. Moreover, in the current situation, because each element of a stimulus is associated with a different task set, an appropriate task set may not be selected based on an exogenous source of information only. It is in these situations when the SAS intervenes to select an appropriate schema. The current results suggest that the SAS may bias one task set so that selection conforms to the order implied by the current task transition in cases in which foreknowledge includes task-identity as well as task transition information. When foreknowledge specifies only task transition and not task identity or when there is no foreknowledge at all, the SAS may not bias a particular task, resulting in no preparation for either task.

The current results can also be accommodated by the adaptive control of thought rational (ACT-R) framework (Anderson, 1993; Anderson \& Lebiere, 1998), which has two layers of control: symbolic level and subsymbolic level. At the symbolic level, contents of declarative knowledge or procedural knowledge may change depending on the current goal. Executive control may be realized by this change at the symbolic level. At the subsymbolic 
level, the access rate of each declarative chunk and procedural production are determined, and this access rate is not under subjective control. Automatic control can be realized by the changes at the subsymbolic level, so that accessing the same chunk or executing the same production may result in priming benefit.

\section{Conclusion}

The current results suggest that task-set reconfiguration is controlled both by an executive mechanism and by a stimulus-driven automatic mechanism. As reflected by the switch cost, intention formation by executive control may be insufficient unless there is external support from the environment. Even when it is obvious which task should be performed and which task should not be performed, as with foreknowledge about task transition and task identity, the not-to-be-performed task may be completely disabled only when there is an external stimulus available to be processed by the to-be-performed task. This process was characterized earlier as a limit on endogenous preparation. What does executive control do? On the basis of current results, it appears that executjve control is responsible for initiating preparation of a new task set but that disengaging from a previous task depends on the operation of automatic, activation-based mechanisms and on the availability of objects to be processed by a new task set. Instantiating goals to form intentions is central to switching tasks (Carlson, 1997; Sohn \& Carlson, 1998) but operates in an informational environment not directly under executive control.

\section{References}

Allport, D. A., Styles, E. A., \& Hsieh, S. (1994). Shifting intentional set: Exploring the dynamic control of tasks. In C. Umilta \& M. Moscovitch (Eds.), Attention and performance XV (pp. 421-452). Cambridge, MA: MTT Press.

Anderson, J. R. (1983). The architecture of cognition. Cambridge, MA: Harvard University Press.

Anderson, J. R. (1993). Rules of the mind. Hillsdale, NJ: Erlbaum.

Anderson, J. R., \& Lebiere, C. (1998). The atomic component of thought. Hillsdale, NJ: Erlbaum.

Baddeley, A. (1986). Working memory. Oxford, England: Oxford University Press.

Carlson, R. A. (1997). Experienced cognition. Hillsdale, NJ: Erlbaum.

Carlson, R. A., \& Lundy, D. H. (1992). Consistency and restructuring in learning cognitive procedural sequences. Joumal of Experimental Psychology: Learning, Memory, and Cognition, 18, 127-141.

De Jong, R. (in press). An intention-activation account of residual switch costs. In S. Monsell \& J. Driver (Eds.), Attention and performance XVIII: Cognitive control. Cambridge, MA: MIT Press.

De Jong, R., Berendsen, E., \& Cools, R. (1999). Goal neglect and inhibitory limitations: Dissociable causes of interference effects in conflict situation. Acta Psychologica, 101, 379-394.

Gopher, D., Armony, L., \& Greenshpan, Y. (1998). Switching tasks and attention policies and the ability to prepare for such shifts. Unpublished manuscript.
Jersild, A. T. (1927). Mental set and shift. Archives of Psychology, Whole No. 89.

Lhermitte, F. (1983). "Utilization behaviour" and its relation to lesions of the frontal lobes. Brain, 106, 237-255.

Logan, G. D. (1985). Executive control of thought and action. Acta Psychologica, 60, 193-210.

Los, S. A. (1996). On the origin of mixing costs: Exploring information processing in pure and mixed blocks of trials. Acta Psychologica, 94, $145-188$.

Meiran, N. (1996). Reconfiguration of processing mode prior to task performance. Journal of Experimental Psychology: Leaming, Memory, and Cognition, 22, 1423-1442.

Meyer, D. E., \& Kieras, D. E. (1997). A computational theory of executive cognitive processes and multiple-task performance: I. Basic mechanisms. Psychological Review, 194, 3-65.

Neely, J. H. (1977). Semantic priming and retrieval from lexical memory: Role of inhibitionless spreading activation and limited capacity attention. Journal of Experimental Psychology: General, 106, 226-254.

Norman, D. A., \& Shallice, T. (1980). Attention to action: Willed and automatic control of behavior. (Technical Report No. 99). Arlington, VA: Center for Human Information Processing.

Posner, M. I., \& Snyder, C. R. R. (1975). Attention and cognitive control. In R. L. Solso (Ed.), Information processing and cognition: The Loyola symposium (pp. 55-85). Hillsdale, NJ: Erlbaum.

Rogers, R. D., \& Monsell, S. (1995). Costs of a predictable switch between simple cognitive tasks. Journal of Experimental Psychology: General, 124, 207-231.

Ruthruff, E., Remington, R., \& Johnston, J. C. (1996, November). Expectancy and repetition and task preparation. Poster presented at the 37th Annual Meeting of the Psychonomic Society, Chicago, IL.

Schneider, W., \& Shiffrin, R. M. (1977). Controlled and automatic human information processing: I. Detection, search, and attention. Psychological Review, 84, 1-66.

Shallice, T. (1994). Multiple levels of control processes. In C. Umilta \& M. Moscovitch (Eds.), Attention and performance XV (pp. 395-420). Cambridge, MA: MTT Press.

Shallice, T., Burgess, P. W., Schon, R., \& Baxter, D. M. (1989). The origins of utilization behavior. Brain, 112, 1587-1597.

Sohn, M.-H., \& Anderson, J. R. (1999). ACT-R does task switching: Effects of foreknowledge and foreperiod on task-switching cost. Poster presented at the 40th Annual Meeting of the Psychonomic Society, Los Angeles, $\mathrm{CA}$.

Sohn, M.-H., \& Carison, R. A. (1998). Procedural framework for simple arithmetic skills. Joumal of Experimental Psychology: Learning, Memory, and Cognition, 24, 1052-1067.

Spector, A., \& Biederman, I. (1976). Mental set and shift revisited. American Journal of Psychology, 89, 669-697.

Strayer, D. L., \& Kramer, A. F. (1994). Strategies and automaticity: I. Basic findings and conceptual framework. Joumal of Experimental Psychalogy: Learning, Memory, and Cognition, 20, 318-342.

Received April 14, 1999

Revision received March 29, 2000

Accepted April 6, 2000 